\title{
Réflexion sur l'évolution des concentrations en radionucléides artificiels dans l'environnement français, les doses associées et l'adaptation des techniques d'analyse aux besoins de la surveillance
}

\author{
PH. RENAUD ${ }^{1}$, R. GURRIARAN ${ }^{2}$
}

(Manuscrit reçu le 28 janvier 2009, accepté le 11 mars 2009)

RÉSUMÉ La concentration de l'environnement français en radionucléides artificiels et les techniques d'analyses ont considérablement évolué au cours des 50 dernières années. Au cours des années 60 et 70, la surveillance effectuée par l'Institut de protection et de sûreté nucléaire (IPSN) et par le service central de protection contre les rayonnements ionisants (SCPRI) permettait de connaître les niveaux des radionucléides artificiels les plus abondants et qui contribuaient de façon prépondérante aux doses reçues par la population française. Depuis les années 80 , avec la fin des essais atmosphériques d'armes nucléaires et la baisse des activités de la plupart des radionucléides concernés, les radionucléides devenus prépondérants en activité et en doses sont : ${ }^{3} \mathrm{H},{ }^{14} \mathrm{C},{ }^{85} \mathrm{Kr}$ et ${ }^{133} \mathrm{Xe}$. Or ces quatre radionucléides ne font aujourd'hui l'objet que d'un nombre très restreint d'analyses dans le cadre de la surveillance effectuée par l'IRSN. La mise en relation des niveaux d'activité des radionucléides constituants le « bruit de fond » de la radioactivité artificielle environnementale, des activités potentiellement ajoutées du fait des rejets des installations nucléaires, des doses correspondantes, ainsi que des limites de détection obtenues au moyens des meilleures techniques disponibles, fournit des éléments de réflexion sur les enjeux de la surveillance radiologique de l'environnement et sur les possibilités d'atteindre les objectifs correspondants. À l'exception de $^{14} \mathrm{C}$ et ${ }^{3} \mathrm{H}$, les activités les plus basses présentes dans l'environnement se trouvent d'ores et déjà au niveau ou en-deçà des limites de détection des techniques de mesure. ${ }^{3} \mathrm{H}$ et ${ }^{14} \mathrm{C}$ sont donc les seuls radionucléides toujours mesurables dans l'environnement et pour lesquels une influence des rejets atmosphériques des installations nucléaires est potentiellement décelable dans tous les milieux.

ABSTRACT Reflection on anthropogenic radionuclide concentrations in the French environment, corresponding doses and adaptation of analysing tools to monitoring needs.

Anthropogenic radioactivity of the French environment and analytical tools, have both significantly evolved during the last 50 years. During the $60 \mathrm{~s}$ and the 70s, the IRSN monitoring allowed knowing activity levels of the most plentiful radionuclides and which provided the main contribution to doses. Since the 80s, the main

1 IRSN, Direction de l'Environnement et de l'Intervention, Service d'Étude et de Surveillance de la Radioactivité dans l'Environnement, Laboratoire d'Étude et Recherches en Milieux Continental et Marin, CE Cadarache, Bâtiment 153, BP 3 , 13115 Saint-Paul-Lez-Durance.

2 Service de Traitement des Échantillons et de Métrologie pour l'Environnement, Laboratoire de Mesure de la Radioactivité dans l'Environnement, CE Cadarache, Bâtiment 153, BP 3, 13115 Saint-Paul-Lez-Durance. 


\begin{abstract}
radionuclides in term of activity and dose $a r e{ }^{3} \mathrm{H},{ }^{14} \mathrm{C},{ }^{85} \mathrm{Kr}$ and ${ }^{133} \mathrm{Xe}$. However, these nuclides are poorly analysed. The relationship between radioactive backgrounds, potentially added activities linked to nuclear releases, corresponding doses, and detection limits using best analytical tools available, provides factors to the radiological monitoring stakes thought. Except for ${ }^{3} \mathrm{H}$ and ${ }^{14} \mathrm{C}$, lowest activities of radionuclides in the environment are still at the level or below detection limits. ${ }^{3} \mathrm{H}$

and ${ }^{14} \mathrm{C}$ are the only ones that can be currently measured and which can label the various environmental compartments around nuclear facilities.
\end{abstract}

Keywords: environmental radioactivity / monitoring / radiological analysis / detection limits

\title{
1. Introduction
}

La concentration de l'environnement français en radionucléides artificiels a considérablement évolué au cours des 50 dernières années. Du milieu des années 50 jusqu'en 1986 et avec des réponses plus ou moins rapides suivant les milieux, cette évolution est allé de pair avec celle de l'air qui a été le principal vecteur des contaminations liées aux retombées des essais atmosphériques d'armes nucléaire et aux retombées de l'accident de Tchernobyl. Depuis le milieu des années 80, ce sont les radionucléides à durée de vie suffisamment longue, accumulés dans les sols, et dans une moindre mesure les sédiments, qui marquent l'ensemble de l'environnement à des niveaux beaucoup plus faibles que lors de la période précédente.

Ce nouveau contexte, mais aussi les attentes de la société, imposent que les stratégies et les techniques mises en œuvre pour la surveillance radiologique évoluent parallèlement (Champion et Peres, 2009).

Cette présentation a pour objectif d'aborder certains aspects plus techniques de cet état des lieux et des axes d'évolution: les radionucléides analysés, leurs niveaux d'activité et les limites de détection atteintes au moyen des meilleurs techniques en usage à l'IRSN, en se focalisant sur l'air et quelques denrées représentatives des différentes catégories d'aliments.

La mise en relation de ces éléments avec d'une part les doses efficaces correspondantes et d'autre part les activités potentiellement ajoutées du fait des rejets des installations nucléaires, permet d'alimenter la réflexion sur certains enjeux de la surveillance radiologique (et de la métrologie associée) et sur les possibilités d'atteindre les objectifs correspondants.

\section{2. Évolution de la radioactivité artificielle de l'environnement français au cours des 50 dernières années et doses correspondantes}

La figure 1 présente l'évolution des activités volumiques des radionucléides artificiels dans l'air depuis 1959. Les données à l'origine de cette figure 
RÉFLEXION SUR L'ÉVOLUTION DES CONCENTRATIONS EN RADIONUCLÉIDES ARTIFICIELS

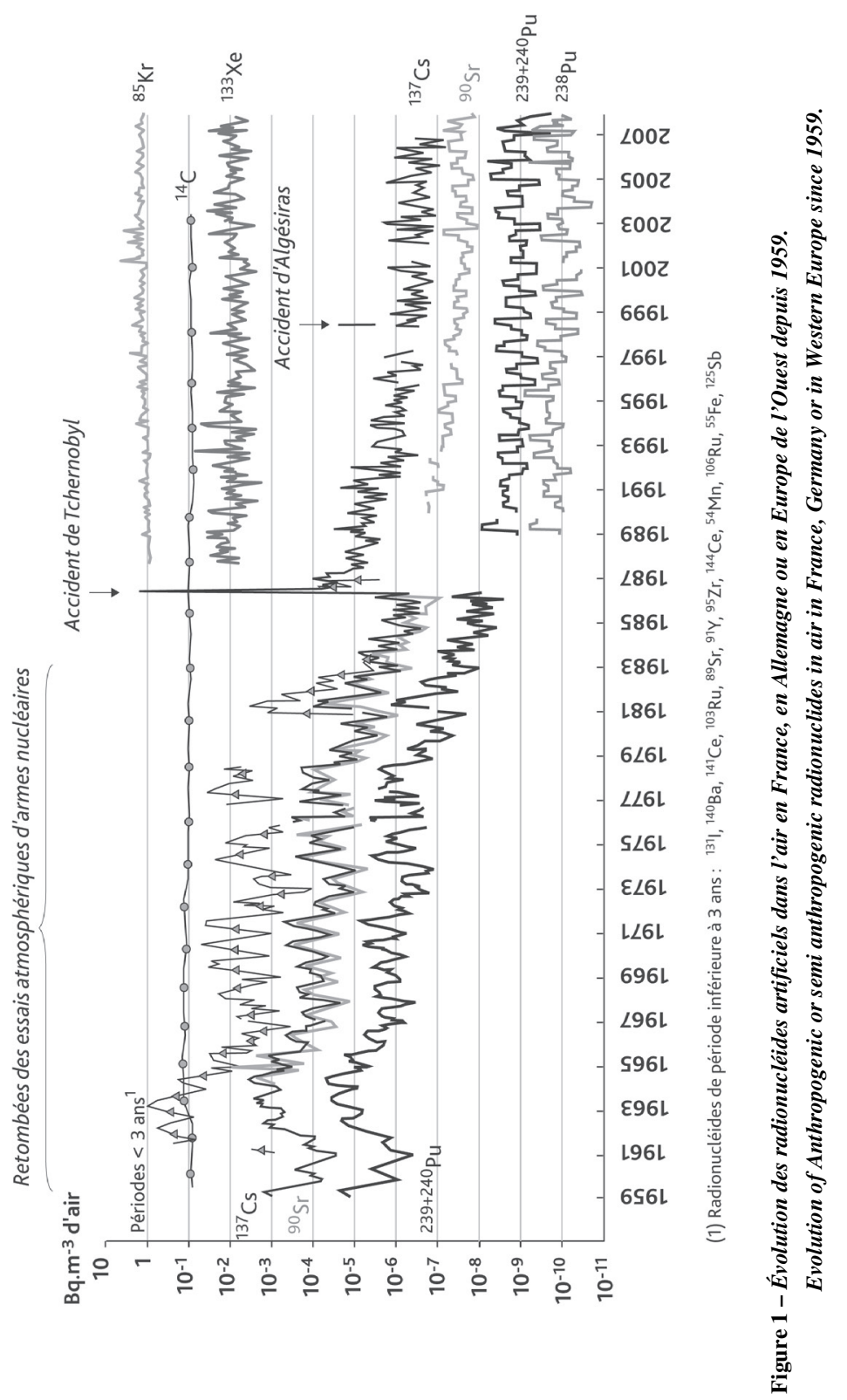

RADIOPROTECTION - VOL. 44 - N² 2 (2009) 


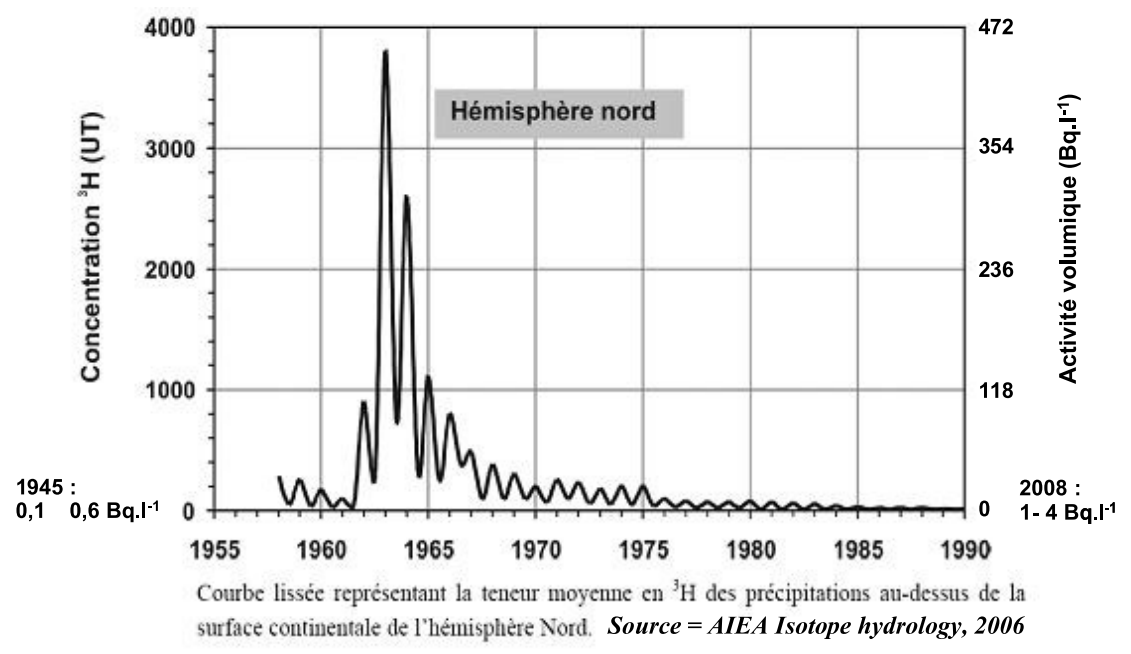

Figure 2-Évolution de la teneur en tritium de l'eau de pluie de 1958 à 1990 exprimée en Unité Tritée (UT) et en Bq $l^{-1}$. Les gammes d'activités correspondant d'une part au bruit de fond naturel cosmogénique antérieur aux essais nucléaires et d'autre part au niveau actuel, ont été ajoutée à la courbe de l'AIEA.

${ }^{3} \mathrm{H}$ in rainwater from 1958 to 1990 , in Tritium Unit and in $\mathrm{Bq} l^{-1}$. Activity ranges linked to natural cosmogenic background and present day levels have been added to the IAEA's figure.

proviennent : du réseau OPERA de l'IPSN puis de l'IRSN (Bouisset et al., 2002) en ce qui concerne les radionucléides à période courte et le ${ }^{137} \mathrm{Cs}$, d'Allemagne (PTB, 2005) pour le ${ }^{85} \mathrm{Kr}$, ${ }^{133} \mathrm{Xe},{ }^{90} \mathrm{Sr}$ et ${ }^{139+240} \mathrm{Pu}$, et de la bibliographie internationale pour le ${ }^{14} \mathrm{C}$ (Roussel-Debet, 2007). En ce qui concerne le tritium, l'AIEA (IAEA/WMO, 2006) fournit une courbe d'évolution de la teneur en tritium de l'eau de pluie de 1958 à nos jours, exprimée en unité tritiée (UT) à laquelle il est possible de faire correspondre une activité volumique en $\mathrm{Bq}^{-1}$ (Fig. 2). L'activité volumique du tritium dans l'air peut être déduite de l'activité volumique de l'eau de pluie pour une humidité absolue moyenne de l'air (masse de vapeur d'eau par volume d'air) et en considérant que l'activité du tritium dans de l'eau de pluie et en équilibre avec celle de la vapeur d'eau. Ainsi, à une activité de $1 \mathrm{~Bq} \mathrm{l}^{-1} \mathrm{~d}$ 'eau de pluie correspond une activité volumique de l'air de l'ordre de $0,01 \mathrm{~Bq} \mathrm{~m}^{-3} \mathrm{~d}^{\prime}$ air.

$\mathrm{Au}$ cours des années 50 et 60 , l'essentiel de la radioactivité artificielle atmosphérique et des denrées, était imputable au tritium et à des produits de fission et d'activation de période courte (moins de 3 ans) issus des retombées des essais d'armes nucléaires : ${ }^{95} \mathrm{Zr},{ }^{144} \mathrm{Ce},{ }^{91} \mathrm{Y},{ }^{106} \mathrm{Ru}$ notamment. En 1963 par exemple, la somme des activités volumiques dans l'air, hors tritium, atteint $1 \mathrm{~Bq} \mathrm{m^{3 }}$ (Renaud et Louvat, 2004) et celle du tritium quelques $\mathrm{Bq} \mathrm{m}^{-3}$ (soit 100 à 1000 fois plus 
élevée que le bruit de fond cosmogénique de ce radionucléide). Cette année-là, la dose efficace à l'adulte, correspondant à ces radionucléides, a été de l'ordre de $50 \mu \mathrm{Sv}$ pour l'inhalation (principalement due aux isotopes du plutonium) et de $70 \mu \mathrm{Sv}$ pour l'exposition externe (principalement due au ${ }^{95} \mathrm{Zr}$ ). En juin 1963, mois durant lequel les retombées des essais nucléaires ont été les plus importantes en France métropolitaine, la contamination des salades a dépassé $500 \mathrm{~Bq} \mathrm{~kg}^{-1}$ (hors tritium et carbone-14), et la dose efficace par ingestion pour un adulte sur l'année a était de l'ordre de $260 \mu \mathrm{Sv}$ (Vray et Renaud, 2004, 2006).

Les activités de ces radionucléides dans l'air ont fortement diminué après 1963 et la contamination des denrées en radionucléides artificiels a diminué de pair. Au cours des années 70, les activités dans l'air ont fluctué avec les bouffées consécutives aux essais nucléaires chinois, avant de décroître significativement à la fin des années 70 et au cours des années 80. À noter que depuis la fin des années 60 , les activités en ${ }^{85} \mathrm{Kr}$, ${ }^{14} \mathrm{C}$ et tritium étaient devenues prépondérantes dans l'air, même si leurs contributions aux doses restaient plus faibles que celle des autres radionucléides.

Les retombées de l'accident de Tchernobyl se sont traduites par une augmentation aussi soudaine que brève des activités dans l'air, principalement de ${ }^{132} \mathrm{Te}$ et ${ }^{131} \mathrm{I}$, dans une moindre de ${ }^{137} \mathrm{Cs}$, ${ }^{134} \mathrm{Cs}$ et de quelques autres produits de fission. Si la somme de leurs activités moyennes sur 10 jours, présentée sur la figure 1 , dépasse à peine $1 \mathrm{~Bq} \mathrm{~m}^{-3}$, les activités quotidiennes observées du $1^{\mathrm{er}}$ au 3 mai ont été plus élevées : jusqu'à $7 \mathrm{~Bq} \mathrm{~m}^{-3}$ à Verdun pour le seul ${ }^{137} \mathrm{Cs}$ (Renaud et al., 2007). Cette bouffée de contaminants atmosphériques a entraîné une augmentation tout aussi soudaine et brève (quelques semaines) de l'activité en ${ }^{131} \mathrm{I}$, ${ }^{137} \mathrm{Cs}$ et ${ }^{134} \mathrm{Cs}$ de denrées comme les légumes et le lait ; plus prolongée mais plus faible pour les viandes et des produits naturels (quasi absence de radionucléides à vie courte et notamment $\mathrm{d}^{131}$ I dans ces produits). Les salades ont été les denrées les plus contaminées avec des maxima de plus de $7000 \mathrm{~Bq} \mathrm{~kg}^{-1}$ (somme des 8 principaux produits de fission) dont près d'un tiers dû à ${ }^{1}{ }^{131}$ I. Les doses efficaces correspondantes ont atteint dans l'Est du pays, $50 \mu \mathrm{Sv}$ pour un adulte par inhalation durant le passage des masses d'air et $300 \mu \mathrm{Sv}$ par ingestion durant l'année 1986.

À partir de 1987, les radionucléides de périodes courtes (moins de 3 ans) provenant des retombées des essais d'armes nucléaires et de l'accident de Tchernobyl, qui étaient les plus abondants dans l'environnement et constituaient depuis le début des années 50 l'essentiel des doses due à la radioactivité d'origine anthropique, ont définitivement «disparu» du milieu atmosphérique et de l'environnement terrestre en général. Dès lors ne subsistent plus dans l'atmosphère que les radionucléides de période moyenne ou longue : ${ }^{14} \mathrm{C},{ }^{3} \mathrm{H},{ }^{137} \mathrm{Cs},{ }^{90} \mathrm{Sr}$ et les isotopes du plutonium. 
Si l'on excepte le ${ }^{137} \mathrm{Cs}$ dont les activités dans l'air ont décru de près d'un facteur 1000 entre 1987 et 1997 , les niveaux de ${ }^{3} \mathrm{H},{ }^{14} \mathrm{C},{ }^{90} \mathrm{Sr}$ et plutonium dans l'air ont lentement décrus pour se stabiliser à partir du début des années 90. Pour ${ }^{3} \mathrm{H}$ et ${ }^{14} \mathrm{C}$, il s'agit d'un retour progressif au bruit de fond d'origine naturel, proche de celui qui prévalait avant les essais d'armes nucléaires ; ce retour au bruit de fond naturel concerne aujourd'hui tous les compartiments de l'environnement.

Pour le ${ }^{137} \mathrm{Cs}$, le ${ }^{90} \mathrm{Sr}$ et les isotopes du plutonium (et leurs descendants, tels que ${ }^{241} \mathrm{Am}$ ), la diminution de leurs activités dans les autres compartiments de l'environnement, et notamment dans les denrées, a été plus lente car liée désormais à celle du sol et non plus à celle de l'air, avec des périodes de décroissance apparente (« effective ») de l'ordre de 4 à 12 ans (sols et denrées) (Roussel-Debet et al., 2007).

\section{La situation actuelle dans l'air}

La figure 3 présente les activités dans l'air observées ces dernières années. La situation actuelle se caractérise par une grande stabilité pour tous les radionucléides artificiels et une nette prédominance de ${ }^{85} \mathrm{Kr},{ }^{3} \mathrm{H},{ }^{14} \mathrm{C}$ et ${ }^{133} \mathrm{Xe}$ au niveau du $\mathrm{Bq} \mathrm{m}{ }^{-3}$ pour le premier et de $0,01 \mathrm{~Bq} \mathrm{~m}^{-3}$ pour les 3 autres. ${ }^{3} \mathrm{H}$ et ${ }^{14} \mathrm{C}$ se rapprochent lentement du bruit de fond naturel cosmogénique : de $10^{-3}$ à $6 \times 10^{-3} \mathrm{~Bq} \mathrm{~m}^{-3}$ pour ${ }^{3} \mathrm{H}$ et de $7 \times 10^{-2}$ à $9 \times 10^{-2} \mathrm{~Bq} \mathrm{~m}^{-3}$ pour ${ }^{14} \mathrm{C}$. Le ${ }^{85} \mathrm{Kr}$ est en légère augmentation dans l'air du fait des rejets des installations de traitement et de recyclage du combustible nucléaire (en tant que gaz rare il n'est pas piégé dans la biosphère), passant de 1 à $1,2 \mathrm{~Bq} \mathrm{~m}^{-3}$ entre 1987 et 2007. Les autres radionucléides artificiels transportés par des aérosols $\left({ }^{137} \mathrm{Cs},{ }^{90} \mathrm{Sr}\right.$ et isotopes du $\left.\mathrm{Pu}\right)$ se situent en-deçà de $1 \mu \mathrm{Bq} \mathrm{m}{ }^{-3}$.

L'usine de La Hague est, parmi les installations nucléaires françaises, celle dont les rejets atmosphériques sont les plus importants avec par ordre décroissant : ${ }^{85} \mathrm{Kr},{ }^{3} \mathrm{H},{ }^{14} \mathrm{C},{ }^{131} \mathrm{I},{ }^{106} \mathrm{Ru},{ }^{125} \mathrm{Sb}$ et ${ }^{137} \mathrm{Cs}$. Les activités volumiques moyennes dans l'air ajoutées par ces rejets au niveau du groupe de référence sont présentées dans la figure 3 (GRNC, 2007). Les activités moyennes attendues en ${ }^{85} \mathrm{Kr}$ du fait des rejets de la Hague, confirmées par des mesures effectuées par l'IRSN et AREVA (Germain et Masson, 2003), sont localement très supérieures au bruit de fond, entre quelques centaines de $\mathrm{Bq} \mathrm{m}^{-3}$, et pouvant ponctuellement atteindre quelques milliers de $\mathrm{Bq} \mathrm{m}{ }^{-3}$. De même, celles de tritium ou de ${ }^{14} \mathrm{C}$ induisent une augmentation mesurable, d'un facteur 4 pour ${ }^{14} \mathrm{C}$ à 10 pour ${ }^{3} \mathrm{H}$ par rapport au bruit de fond cosmogénique de ces radionucléides. En revanche, celles des autres radionucléides se situent le plus souvent en dessous des limites de détection, hormis sporadiquement dans l'environnement très immédiat du site. 


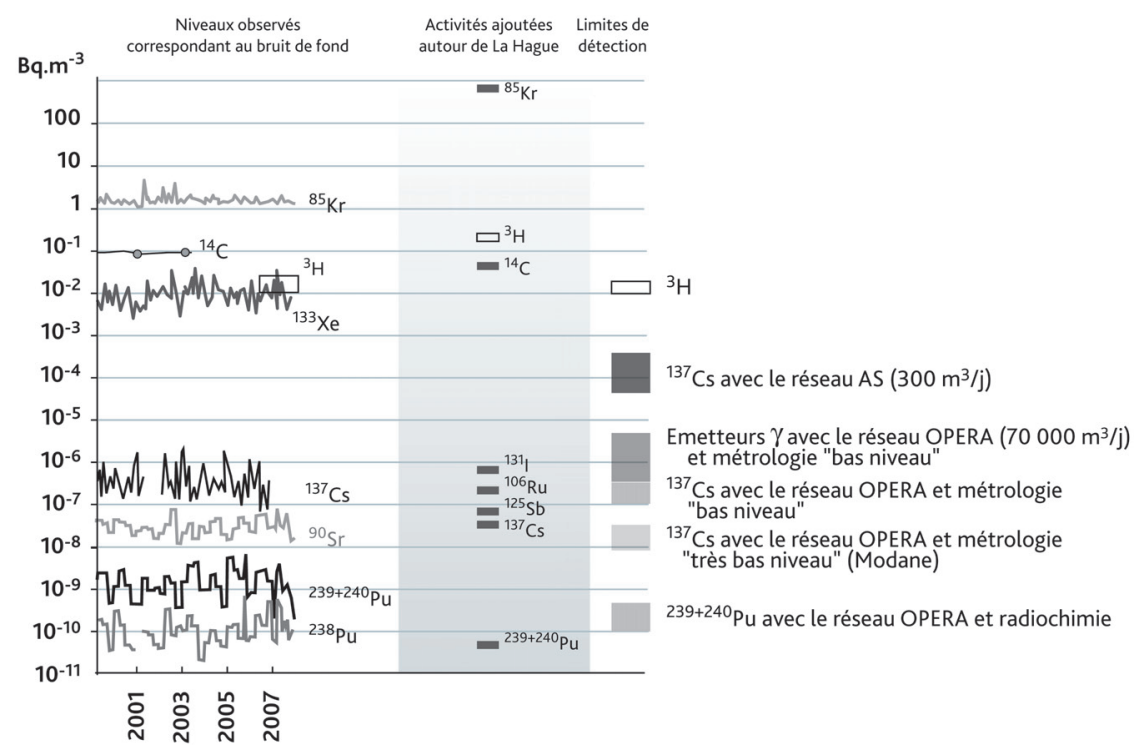

Figure 3-Activités volumiques actuelles des radionucléides artificiels ou semi artificiels dans l'air, activités moyennes potentiellement ajoutées par les rejets de La Hague au niveau du groupe de référence, limites de détection de différents systèmes d'acquisition.

Present volumic activities of anthropogenic or semi anthropogenic radionuclides, mean potentially added activities by La Hague releases for the reference group, detection levels of various acquiring systems.

Excepté le tritium autour de certaines installations comme Valduc ou Marcoule, les activités en radionucléides artificiels potentiellement ajoutées dans l'air du fait des rejets des autres installations nucléaires sont inférieures, voire très inférieures à celles attendues ou mesurées autour de La Hague. Par ailleurs, aux observations effecuées autour de La Hague et liées à ses rejets, est associé un impact dosimétrique inférieur à $20 \mu \mathrm{Sv} \mathrm{an}^{-1}$, tous radionucléides confondus, pour les populations riveraines susceptibles d'être les plus exposées (GRNC, 2007).

La figure 3 permet également une comparaison avec les limites de détections actuellement obtenues par différents systèmes d'acquisition mis en œuvre par l'IRSN. Il apparaît que les activités de ${ }^{137} \mathrm{Cs},{ }^{90} \mathrm{Sr}$ et des isotopes du plutonium se situent d'ores et déjà au niveau des limites de détection des meilleurs techniques de prélèvement et d'analyse disponibles: des échantillons d'aérosols correspondant à la filtration de $70000 \mathrm{~m}^{3}$ soit 10 jours de prélèvement d'une station du réseau OPERA, un comptage gamma sur 2 jours et une métrologie à bas bruit de fond au Laboratoire de mesure de la radioactivité de l'environnement de l'IRSN d'Orsay (Bouisset et al., 2002). Pour diminuer encore ces limites de 
détection et poursuivre ce suivi dans la prochaine décennie, l'IRSN investit dans des stations à débit de prélèvement 2 fois supérieur. Outre continuer à disposer d'un référentiel et poursuivre des chroniques, supports aux recherches sur les phénomènes de transport atmosphérique et d'événements naturels, ces stations peuvent déceler des rejets accidentels discrets ou dont la source est éloignée, comme l'incendie du silo à La Hague (1981), des rejets anormaux (ruthénium à La Hague - 1997 et 2002, l'incident d'Algésiras - 1998). Par ailleurs, en cas d'événement majeur, elle permettrait de caractériser le retour à la normale dans le temps et à distance de l'installation. Compte tenu des contraintes associées à l'obtention de données à de si bas niveaux, il n'est pas envisageable d'entretenir plus d'une douzaine de stations de ce type. Ces quelques stations viennent néanmoins compléter un réseau plus dense (réseau AS, de l'ordre de 60 stations), orienté essentiellement vers la surveillance des rejets anormaux des installations nucléaires, et dont les performances sont aussi en cours d'amélioration. Elles permettraient de diminuer d'un facteur 50 à 100 les limites de détection actuelles de ce réseau, se rapprochant ainsi des limites de détection du réseau OPERA actuel.

En ce qui concerne le tritium, le suivi effectué dans l'eau de pluie autour de certains sites nucléaires dans le cadre de la surveillance autour de certains sites nucléaire, présente deux inconvénients, d'une part établir la relation entre l'activité de l'air et celle de l'eau de pluie nécessite, comme indiqué précédemment, des hypothèses, et d'autre part cette surveillance n'est pas permanente ne se faisant que lors des pluies. Pour lever cette difficulté, le déploiement de dispositifs de collecte de la vapeur d'eau tritiée (barboteur) est à favoriser. Les performances de ce type de station permettent de révéler toute anomalie du bruit de fond. La seule station implantée en France dans le cadre de la surveillance de l'environnement assurée par l'IRSN (hormis celles déployées par les exploitants nucléaires) ; située sous les vents dominants de l'installation de Valduc, montre des concentrations en tritium sous forme de vapeur d'eau comprise entre 0,5 et quelques $\mathrm{Bq} \mathrm{m}^{-3}$ sur toute l'année (IRSN, 2007).

Aucun dispositif pérenne n'est actuellement déployé pour la surveillance du ${ }^{14} \mathrm{C}$ en France. Néanmoins, il convient de noter que pour ce radionucléide, et dans une certaine mesure pour ${ }^{3} \mathrm{H}$ également, toute sortie anormale du bruit de fond atmosphérique peut être révélée bien qu' a posteriori, au travers de prélèvements périodiques de végétaux intégrateurs biologiques (Roussel-Debet, 2007, 2008).

Les doses efficaces pour un adulte correspondant à ces activités du bruit de fond sont de l'ordre du $\mathrm{nSv}$ an ${ }^{-1}$ pour ${ }^{85} \mathrm{Kr}$ (par exposition externe), ${ }^{133} \mathrm{Xe}$ (exposition externe) et ${ }^{14} \mathrm{C}\left(5 \mathrm{nSv} \mathrm{an}^{-1}\right.$ par inhalation). À noter que les doses dues au ${ }^{85} \mathrm{Kr}$ autour de la Hague peuvent atteindre quelques $\mu \mathrm{Sv} \mathrm{an}^{-1}$. Les doses dues 
aux autres radionucléides, fixés sur des aérosols $\left({ }^{137} \mathrm{Cs}\right.$, isotopes du $\mathrm{Pu}$ ) sont inférieures au $\mathrm{nSv} \mathrm{an}^{-1}$. Pour mémoire la dose moyenne par inhalation en France due au radon et ses descendants est de l'ordre du $\mathrm{mSv}^{-1}$ et peut atteindre quelques dizaines de $\mathrm{mSv} \mathrm{an}^{-1}$ dans certaines régions.

\section{La situation actuelle dans les denrées}

La figure 4 présente les gammes d'activités observées dans quelques denrées pour les cinq principaux radionucléides artificiels. Les données à l'origine de ce graphique ont été acquises par l'IPSN puis l'IRSN au cours des 8 dernières années, en différents points du territoire, soit dans le cadre du suivi radioécologique effectué à la demande d'EDF autour des installations électronucléaires, soit dans le cadre de la surveillance de l'environnement, notamment via le réseau d'observatoires OPERA, ou encore lors d'études ponctuelles menées à des fins de recherche. Les barres figurant les gammes de valeur sont délimitée par des paramètres statistiques : moyenne arithmétique, quartiles 1 et 3 qui délimitent la couleur pleine, valeurs minimales et maximales qui limitent les dégradés. Lorsque trop peu de données sont disponibles le dégradé se fait immédiatement de part et d'autre de la moyenne jusqu'aux valeurs minimales et maximales.

Les activités très variables s'étalent sur près de 7 décades. Ainsi, les activités de ${ }^{14} \mathrm{C}$, les plus élevées, comprises le plus souvent entre $10 \mathrm{~Bq} \mathrm{~kg}^{-1}$ et $100 \mathrm{~Bq} \mathrm{~kg}^{-1}$, sont 10000 à plus de 1 million de fois supérieures à celles de ${ }^{239+240} \mathrm{Pu}$. Ces dernières sont le plus souvent inférieures à $1 \mathrm{mBq} \mathrm{kg}^{-1}$.

De même, les activités de ${ }^{137} \mathrm{Cs},{ }^{90} \mathrm{Sr}$ et ${ }^{239+240} \mathrm{Pu}$ mesurées sur l'ensemble du territoire couvrent chacune 2 décades sans qu'il soit possible de discriminer statistiquement d'effet régional. L'origine des radionucléides artificiels et les transferts radioécologiques expliquent cette variabilité ${ }^{3}$.

Les gammes d'activités restituées sur ce graphique sont représentatives du «bruit de fond » en France et plus largement en Europe de l'Ouest pour les radionucléides considérés. Ce bruit de fond provient de la rémanence des retombées des essais atmosphériques d'armes nucléaires et de l'accident de Tchernobyl. Sous l'influence des rejets industriels de certaines installations nucléaires, des activités plus élevées peuvent être mesurées. C'est le cas notamment des activités de tritium autour de l'usine de La Hague ou du centre de recherche de Valduc.

\footnotetext{
3 Lors des retombées des retombées des essais nucléaires et dans l'année suivant l'accident de Tchernobyl, une variabilité régionale a pu clairement être mise en évidence. Cette variabilité régionale était liée à celle des dépôts par transfert foliaire direct. Aujourd'hui, la variabilité « radioécologique » liée au devenir des radionucléides dans les sols (lessivage, migration vers la profondeur, biodisponibilité), à l'intensité du transfert racinaire, aux pratiques agricoles et d'élevage, masque le plus souvent la variabilité régionale initiale (des dépôts radioactifs).
} 


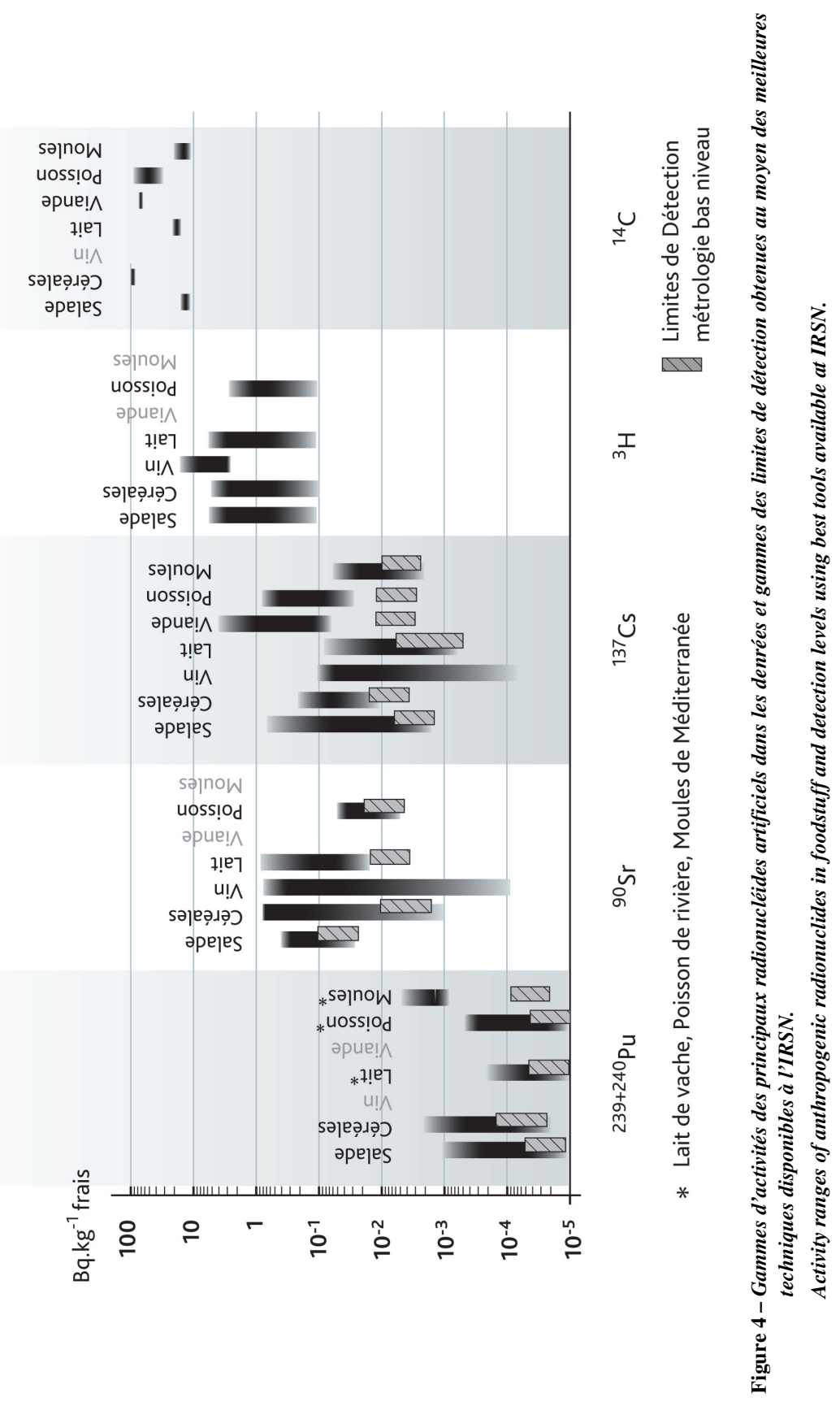


La figure 5 montre qu'hormis pour le tritium et le ${ }^{14} \mathrm{C}$, aucun marquage de l'environnement terrestre par les rejets atmosphériques direct de l'usine de La Hague n'est décelable dans les denrées. En effet, pour les radionucléides appartenant au bruit de fond de la radioactivité artificielle de l'environnement comme ${ }^{137} \mathrm{Cs},{ }^{90} \mathrm{Sr}$ et les isotopes du plutonium, la rémanence des retombées anciennes est nettement plus élevée et masque les activités attendues du fait des rejets atmosphériques.

Pour les autres radionucléides, comme ${ }^{131} \mathrm{I}$ ou ${ }^{106} \mathrm{Ru}$, qui figurent parmi les radionucléides les plus abondants dans les rejets de La Hague, les activités attendues sont théoriquement en-deçà des limites de détection actuelles. Toutefois, des conditions météorologiques particulières lors d'un rejet peuvent amener à des activités avoisinant, voire dépassant les limites de détection. Dans le cas du ${ }^{14} \mathrm{C}$, les activités ajoutées du fait des rejets de la Hague, exprimées en $\mathrm{Bq} \mathrm{kg}^{-1}$, sont masquées par le bruit de fond, mais il existe pour ce radionucléide un indicateur plus sensible et plus discriminant : l'activité spécifique exprimée en $\mathrm{Bq}$ de ${ }^{14} \mathrm{C}$ par kilogramme de carbone élémentaire. L'obtention d'un nombre important de données autour des centres nucléaires de production électrique (CNPE) a permis de révéler une augmentation statistiquement significative de seulement $3 \mathrm{~Bq}$ de ${ }^{14} \mathrm{C}$ par kg de carbone élémentaire en zones influencées par les rejets pour un bruit de fond de $243 \mathrm{~Bq} \mathrm{~kg}^{-1}$ de C (Roussel-Debet et al., 2006).

La plupart des autres installations nucléaires françaises, notamment les CNPE, sont beaucoup moins susceptibles de marquer l'environnement par leurs rejets atmosphériques, hormis en tritium et ${ }^{14} \mathrm{C}$. Par ailleurs, un rejet accidentel de faible amplitude peut être décelable dans l'air sans l'être dans les denrées. Cela a été le cas du rejet accidentel de ${ }^{137} \mathrm{Cs}$ provenant d'Algésiras. Le recours à des bioindicateurs comme des mousses terrestres, des lichens ou des feuilles d'arbres, présente alors au moins deux intérêts : d'une part une meilleure sensibilité vis-àvis d'une augmentation normale ou non de l'activité atmosphérique et de ses retombées au sol, complémentairement aux prélèvements d'air, et d'autre part une intégration sur la durée souhaitée qui permet de déceler un événement survenant entre deux prélèvements.

\section{5. Évolution des techniques d'analyses, possibilités actuelles et perspectives}

Depuis les années 60 et jusqu'à aujourd'hui, l'amélioration des techniques utilisées pour prélever, traiter, conditionner et analyser les échantillons, a permis de suivre la diminution des teneurs en radionucléides artificiels dans l'environnement. Les améliorations ont concerné tous les maillons de la chaîne 

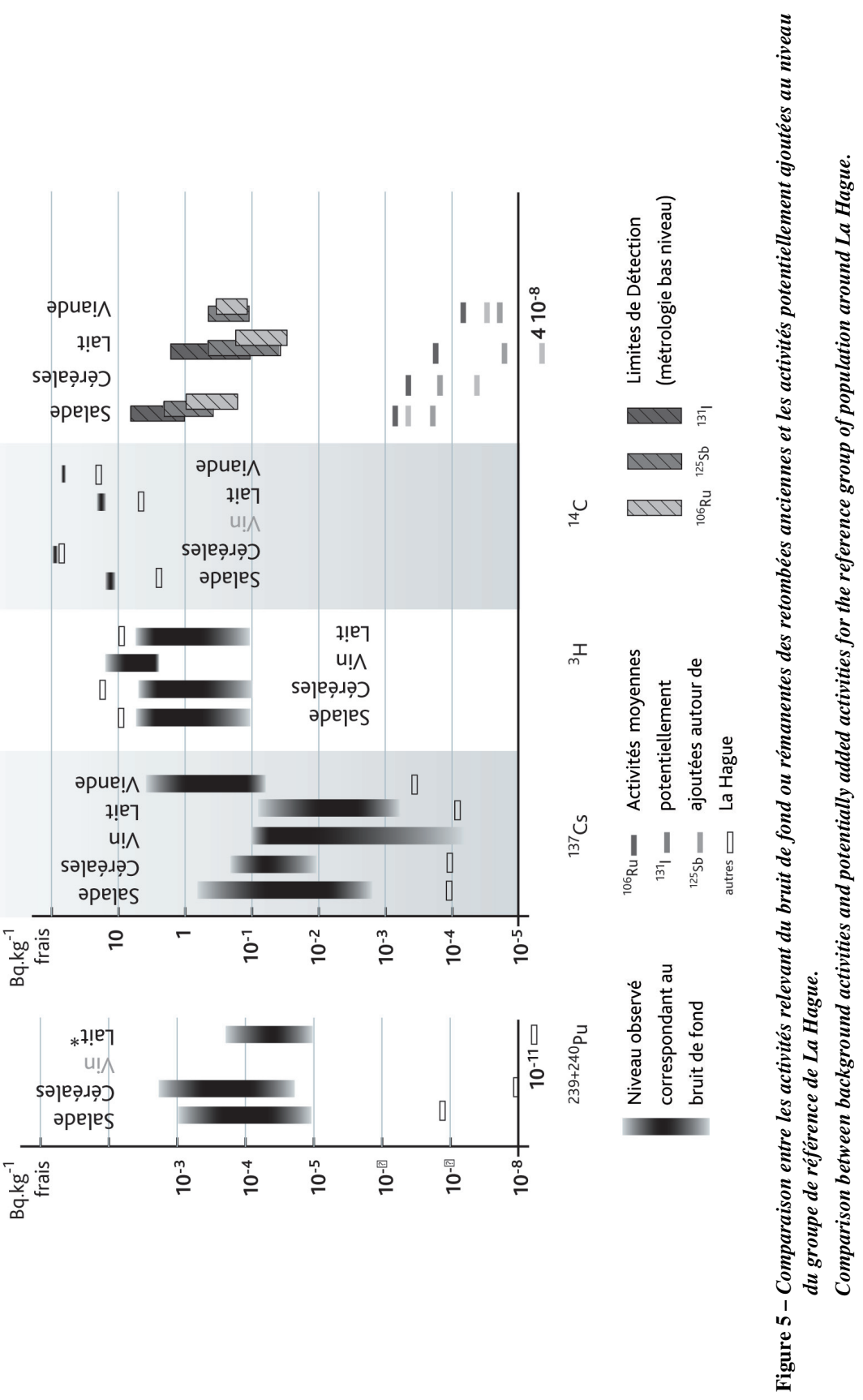
d'acquisition, du prélèvement au dépouillement des spectres, avec comme objectif l'augmentation du signal émis par l'échantillon et la diminution du bruit de fond.

Parmi ces améliorations on peut citer l'augmentation des volumes/masses prélevés, la concentration maximale avec un minimum d'altération par incinération et lyophilisation, l'amélioration de la relation entre le détecteur et l'échantillon (géométrie), le développement progressif du parc de détecteurs germanium, la pureté et la taille des détecteurs $\left(1 \mathrm{~cm}^{3}\right.$ en $1968,960 \mathrm{~cm}^{3}$ aujourd'hui), les améliorations de l'électronique et des logiciels de traitement des spectres, la diminution du bruit de fond (détecteurs bas bruit de fond, système anti Compton et veto cosmique), le recours à la radiochimie pour les analyses d'émetteurs bêta et alpha. Aujourd'hui, obtenir les limites indiquées dans la figure 3 pour un émetteur gamma nécessite de prélever quelques dizaines de kilogrammes de denrées, de nettoyer méticuleusement l'échantillon, de le sécher durant quelques jours en étuve, de l'incinérer durant 2 jours, de l'homogénéiser, de le broyer et le tasser dans la géométrie la plus adaptée, avant un comptage sur 1 journée en utilisant les dispositifs métrologiques précédemment cités. Obtenir ces limites de détection pour un émetteur alpha nécessite, après séchage d'un échantillon de 50 à $200 \mathrm{~g}$, une extraction sélective des atomes durant 3 à 4 semaines de radiochimie (minéralisation, co-précipitation et purification sur colonne), l'électrodéposition des atomes du radionucléide considéré, puis un comptage durant 2 semaines.

Bien que ces techniques très contraignantes soient les plus performantes aujourd'hui, la figure 3 montre que les activités les plus basses dans les denrées (hors ${ }^{14} \mathrm{C}$ ) sont d'ores et déjà au niveau ou au-dessous des limitent de détection qu'elles confèrent. La diminution constatée de ces activités, avec des périodes beaucoup plus courtes que les périodes radioactives (de 4 à 12 ans pour ${ }^{137} \mathrm{Cs}$ par exemple), fait que le nombre d'analyses fournissant des résultats non significatifs (inférieurs aux limites de détection) va inéluctablement augmenter dans les années à venir.

En ce qui concerne les spectrométries alpha (pour les isotopes du $\mathrm{Pu}$ notamment) et bêta ( ${ }^{90} \mathrm{Sr}$ notamment), les possibilités de diminuer les limites de détection par une augmentation des masses prélevées va se heurter à une augmentation des coûts de prélèvement et de prétraitement (capacité d'incinération) et à la capacité de traitement radiochimique actuellement déterminée pour une masse maximale de $300 \mathrm{~g}$ de cendres par échantillon.

Si le bruit de fond en ${ }^{3} \mathrm{H}$ est encore mesurable aujourd'hui avec les méthodes de combustion et synthèse par benzène et la méthode par accélération, le bruit de fond cosmogénique situé à des niveaux 2 à 10 fois inférieurs restera accessible par la technique utilisant la mesure du descendant du ${ }^{3} \mathrm{H}:{ }^{3} \mathrm{He}$. 
Pour le ${ }^{14} \mathrm{C}$, le problème n'est pas la limite de détection mais l'incertitude sur le résultat de mesure. Les meilleures techniques d'analyses actuelles (par benzène ou par accélération), fournissent des incertitudes de l'ordre de $20 \mathrm{~Bq} \mathrm{~kg}^{-1}$ de C. Cette incertitude, actuellement incompressible, n'est pas suffisante pour déceler un marquage léger par une installation (tel qu'attendu dans le cas de rejets normaux) à partir de quelques échantillons. Pour conclure sur une éventuelle influence des rejets, il est nécessaire d'opposer statistiquement deux populations d'échantillons, l'une non influencée et l'autre potentiellement influencée, et donc de disposer d'un nombre conséquent de mesures sur chaque site. La capacité annuelle de l'IRSN pour ces deux types d'analyse (par benzène et par accélération) est théoriquement de 300 , ce qui est très faible au regard des enjeux lié au ${ }^{14} \mathrm{C}$ en matière de surveillance : le radionucléide artificiel le plus abondant et le premier contributeur à la dose par ingestion.

\section{Doses par ingestion unitaire correspondant aux activités artificielles environnementales}

La figure 6 présente les doses efficaces correspondant à l'ingestion de 1 kilogramme (ou litre) de chacune des denrées concernées par un adulte. Elles ont été obtenues en multipliant les activités massiques ou volumiques des denrées par le facteur de dose par ingestion du radionucléide considéré (EURATOM, 1996) fournit dans (www.irsn.ecrin.org). Dans le cas du ${ }^{3} \mathrm{H}$, pour lequel deux facteurs de doses différents sont à considérer, l'un pour le tritium libre et l'autre pour le tritium lié, un facteur de dose pondéré, spécifique à chaque denrée a été estimé.

Les doses efficaces par ingestion unitaire sont très faibles, toujours inférieures à $1 \mu \mathrm{Sv} \mathrm{kg}{ }^{-1}$ et essentiellement entre $10 \mathrm{pSv} \mathrm{kg}^{-1}$ et $10 \mathrm{nSv} \mathrm{kg}^{-1}$. Une estimation grossière en considérant une consommation de denrée de $500 \mathrm{~kg} \mathrm{an}^{-1}$, conduit à une dose annuelle correspondante de $5 \mathrm{nSv}$ an $^{-1}$ à $5 \mu \mathrm{Sv} \mathrm{an}^{-1}$. Ces doses unitaires sont moins dispersées en fonction des radionucléides que ne l'étaient les activités massiques. Ainsi, bien que les activités massiques de plutonium soient faibles, la contribution de ce radionucléide aux doses par ingestion n'est pas négligeable. À l'inverse, malgré des activités plus élevées, le tritium ne contribue pas plus à la dose par ingestion que le plutonium. La plus forte contribution à la dose efficace par ingestion reste le ${ }^{14} \mathrm{C}$. Sans événement marquant, les contributions du ${ }^{137} \mathrm{Cs}$, $\mathrm{du}{ }^{90} \mathrm{Sr}$ et des isotopes du plutonium continueront à diminuer avec les activités massiques et celle du ${ }^{14} \mathrm{C}$ augmentera au moins d'autant.

L'évaluation de cette gamme de dose annuelle est le résultat de l'utilisation des meilleures techniques métrologiques disponibles, sans lesquelles ce résultat ne pourrait être que le résultat d'un calcul basé sur les teneurs dans les sols ou sur des données bibliographiques. 


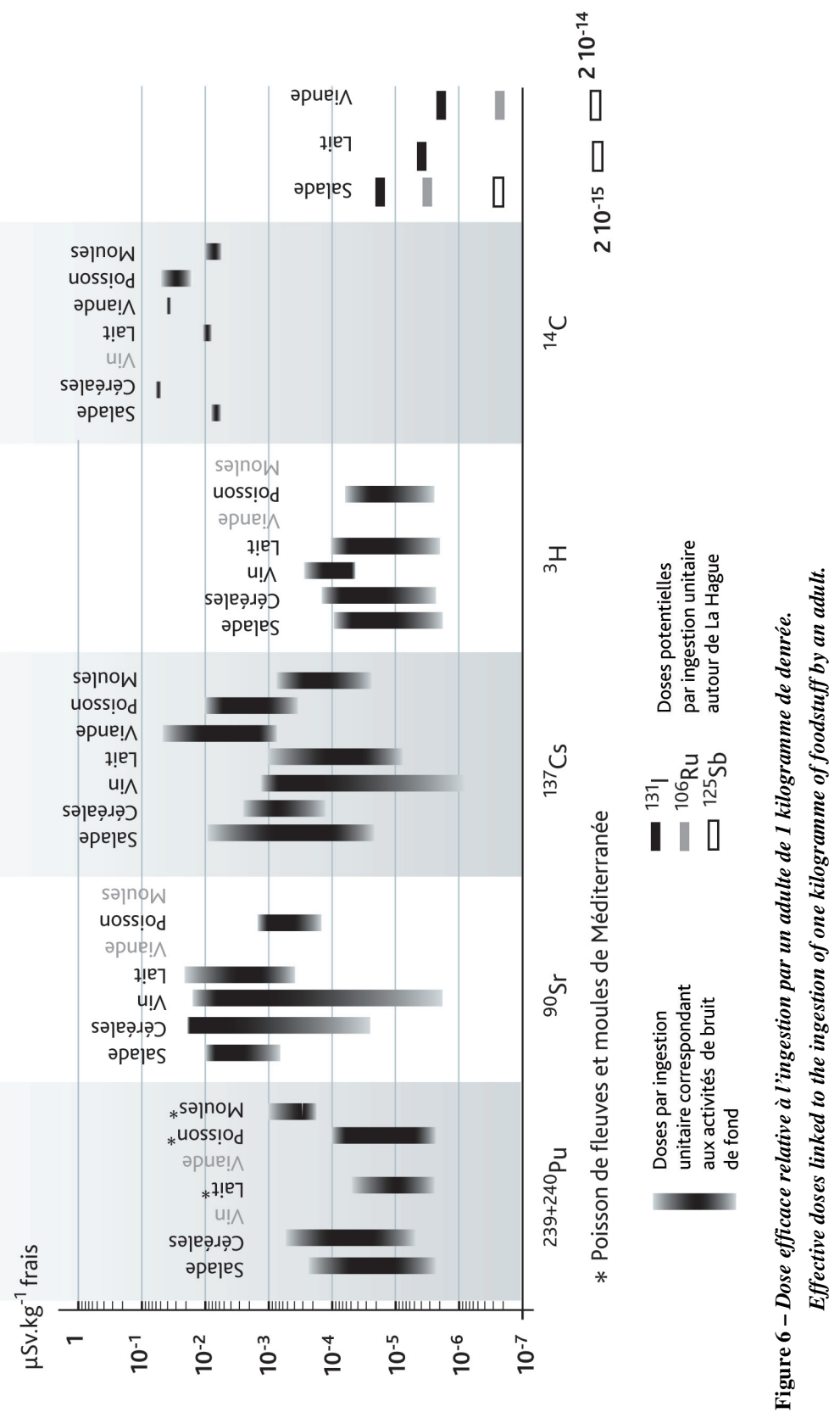




\section{Conclusion}

Au cours des années 60 et 70, la surveillance effectuée par l'IPSN et le SCPRI était pertinente en ce qui concerne les radionucléides analysés. Aussi bien dans l'air que dans les denrées, il s'agissait des radionucléides artificiels les plus abondants et qui contribuaient très majoritairement aux doses: des produits de fission ou d'activation provenant des retombées des essais atmosphériques d'armes nucléaires.

Depuis les années 80, avec la diminution rapide de leurs activités, les radionucléides devenus prépondérants en activité et/ou en doses sont : ${ }^{3} \mathrm{H},{ }^{14} \mathrm{C}$, ${ }^{85} \mathrm{Kr},{ }^{133} \mathrm{Xe}$, dans l'air, et ${ }^{3} \mathrm{H}$ et ${ }^{14} \mathrm{C}$ dans les denrées. Or ces quatre radionucléides ne font l'objet que d'une surveillance très limitée par l'IPSN en milieu atmosphérique, voir nulle pour les ${ }^{85} \mathrm{Kr}$ et ${ }^{133} \mathrm{Xe}$. Cette surveillance se résume à quelques études spécifiques autour de La Hague, une station de prélèvement en continue de tritium atmosphérique sous les vents dominants du site de Valduc, complétée par des stations de suivi d'eau de pluie, et d'un nombre assez restreint d'analyses en ${ }^{14} \mathrm{C}$ dans des indicateurs biologiques (souvent des denrées).

Depuis les années 60 et jusqu'à aujourd'hui, l'amélioration des techniques utilisées pour prélever, traiter, conditionner et analyser les échantillons, a permis de suivre la diminution des teneurs en radionucléides artificiels dans l'environnement. Les améliorations ont concerné tous les maillons de la chaîne d'acquisition, du prélèvement au dépouillement des spectres.

À l'exception de ${ }^{14} \mathrm{C}$ et ${ }^{3} \mathrm{H}$, les activités les plus basses présentes dans l'environnement se trouvent d'ores et déjà au niveau ou en-deçà des limites de détection obtenues grâce aux meilleures techniques disponibles à l'IRSN. Ces activités continueront par ailleurs de baisser dans les années à venir avec des périodes beaucoup plus courtes que la décroissance radioactive.

Par ailleurs, pour les radionucléides artificiels autres que ${ }^{14} \mathrm{C}$ ou ${ }^{3} \mathrm{H}$, les activités ajoutées par les rejets sont, soit masquées par la rémanence des retombées anciennes, soit inférieures aux limites de détection, aussi bien dans les denrées que dans les bio indicateurs.

Néanmoins, l'accident de Tchernobyl et d'autres évènements de moindre ampleur ont montré l'intérêt qu'il y avait à acquérir des résultats sur certains produits de fission dans l'air ou dans les denrées de manière à disposer de niveaux de référence en cas de rejet accidentel.

${ }^{3} \mathrm{H}$ et ${ }^{14} \mathrm{C}$ sont donc les seuls radionucléides toujours mesurables dans l'environnement et pour lesquels une influence des rejets atmosphériques des 
installations nucléaires est potentiellement décelable dans tous les milieux, notamment pour ${ }^{14} \mathrm{C}$ au moyen de l'activité spécifique $\left(\mathrm{Bq} \mathrm{kg}^{-1}\right.$ de carbone élémentaire). Les deux meilleures techniques disponibles pour mesurer ${ }^{14} \mathrm{C}$ permettent même de conclure sur de très faibles influences dès lors que l'on dispose d'un nombre de mesure suffisant pour mener une analyse statistique.

Le milieu fluvial se distingue par le fait que l'influence des rejets liquides des installations nucléaires est visible dans la plupart des indicateurs pour quelques radionucléides : ${ }^{110 \mathrm{~m}} \mathrm{Ag},{ }^{60} \mathrm{Co},{ }^{58} \mathrm{Co}$ et ${ }^{54} \mathrm{Mn}$. Il en est de même en milieu marin dans la zone relativement étendue, influencée par le rejet de La Hague.

La surveillance de l'environnement et notamment des denrées, ne permet pas, hormis pour ${ }^{3} \mathrm{H}$ et ${ }^{14} \mathrm{C}$ de contrôler le respect des autorisations de rejets ou de déceler un éventuel rejet accidentel de faible amplitude. Les activités attendues dans l'environnement étant très inférieures aux limites de détection, seule la surveillance des activités des effluents rejetés par les installations nucléaires permet de répondre à cet objectif.

Dans quelques années et sauf à y consacrer des moyens très importants au regard des enjeux de radioprotection, ces techniques ne permettront plus de connaître le bruit de fond en radionucléides rémanents des retombées anciennes.

À moyen termes, l'objectif de la surveillance environnementale restera de s'assurer que les doses liées aux activités environnementales sont extrêmement faibles. Il appartient aux autorités et aux experts de fixer ces niveaux de doses et donc l'importance du challenge métrologique à relever.

Remerciements. Marcel Morello et Patrick Bouisset pour les aspects métrologiques; Caroline Ringeard, Myriam Oddon et Alain Després pour les données du GRNC autour de La Hague; Michel Masson et Denis Marot pour les niveaux autour de La Hague; Sylvie Roussel-Debet, Gilles Gontier, Olivier Masson, Christelle Antonilli, Céline Duffa, Fédérique Eyrolle, Laurent Pourcelot et Hervé Thébault du LERCM.

\section{RÉFÉRENCES}

Bouisset P., Barker E., Masson O., Gurriaran R., Cagnat X., Mekhlouche D., Aubry S., Hadjaj M., Saey L. (2002) Concentration de ${ }^{137} \mathrm{Cs}$ et de ${ }^{7} \mathrm{Be}$ dans les aérosols en France métropolitaine et à Tahiti de 1959 à 2002, Radioprotection 39(2), 367-381.

Champion D., Peres J.-M. (2009) L'IRSN et la surveillance de la radioactivité de l'environnement en France : état des lieux et perspectives, Radioprotection 44(2), 217-240.

EURATOM (1996) Directive 96-29 du Conseil du 13 mai 1996 fixant les normes de base relatives à la protection sanitaire de la population et des travailleurs contre les dangers résultants des rayonnements ionisants. 
PH. RENAUD, R. GURRIARAN

Germain P., Masson M. (2003) Étude du comportement des radionucléides rejetés par l'usine de La Hague, Rapport IRSN/DPRE/SERNAT 2003-01.

GRNC (2007) Groupe Radioécologie Nord-Cotentin, Rapport de synthèse et rapport détaillé 2005 : Appréciation par le GRNC de l'estimation des doses présentées dans le rapport annuel de surveillance de l'environnement d'AREVA-NC La Hague.

IAEA/WMO (2006) Global Network of Isotopes in Precipitation. The GNIP Database; available at: http://isohis.iaea.org.

IRSN (2007) Bilan de l'état radiologique de l'environnement français en 2007, synthèse des résultats des réseaux de surveillance de l'IRSN. Rapport des résultats des réseaux de surveillance, Rapport IRSN/DEI/SESURE 2008-48.

PTB (2005) Physikalisch-Technische Bundesanstalt, Radionucides in ground-level air in Braunschweig, Report of the PTB Trace Survey Station from 1998 to 2003.

Renaud Ph., Louvat D. (2004) Magnitude of fission product depositions from atmospheric nuclear weapon test fallout in France, Health Phys. 86(4), 353-358.

Renaud Ph., Champion D., Brenot J. (2007) Les retombées radioactives de l'accident de Tchernobyl sur le territoire français; conséquences environnementales et exposition des personnes. Tec\&doc Lavoisier, collection Sciences et Techniques. 190p.

Roussel-Debet S. (2007) Evaluation of ${ }^{14} \mathrm{C}$ doses since the end of the 1950s in metropolitan France, Radioprotection 42(3), 297-313.

Roussel-Debet S. (2008) Interprétation des résultats des mesures de carbone-14 réalisées sur des échantillons végétaux prélevés dans l'environnement de l'usine SOCATRI en juillet et août 2008, Rapport IRSN/DEI/SESURE 2008-52.

Roussel-Debet S., Gontier G., Siclet F., Fournier M. (2006) Distribution of carbon-14 in the terrestrial environment close to French nuclear power plants, J. Environ. Radioact. 79, 171-185.

Roussel-Debet S., Renaud Ph., Métivier J.M. (2007) ${ }^{137}$ Cs in French soils: deposition patterns and 15-year evolution, Sci. Tot. Environm. 374, 388-398.

Vray F., Renaud Ph. (2004) Contamination de la chaîne alimentaire par les produits de fissions émis lors des essais d'armes nucléaires, Rapport IRSN/DEI/SESURE 2004-19.

Vray F., Renaud Ph. (2006) Conséquences dosimétriques des essais aériens d'armes nucléaires en France métropolitaine, Période 1961-1978, Rapport IRSN/DEI/SESURE 2006-02. 